\title{
Simulation of boundary slip on a liquid-solid surface based on the lattice Boltzmann method
}

\author{
Kai Wang ${ }^{\mathrm{a}}$, Yao Zhang ${ }^{\mathrm{a}}$, Yang $\mathrm{Yu}^{\mathrm{a}}$, Guoxiang Hou ${ }^{\mathrm{a}, *}$, Feng Zhou ${ }^{\mathrm{b}}$, Yang $\mathrm{Wu}^{\mathrm{b}}$ \\ a School of Naval Architecture and Ocean Engineering, Huazhong University of Science and Technology, \\ Wuhan, 430074, China \\ b State Key Laboratory of Solid Lubrication, Lanzhou Institute of Chemical Physics, \\ Chinese Academy of Sciences, Lanzhou 730000 China
}

*Corresponding author, e-mail: houguoxiang@163.com

Received 2 Sep 2014

Accepted 16 Apr 2015

\begin{abstract}
Boundary slip phenomena on a superhydrophobic surface at a mesoscopic scale are investigated. Using the lattice Boltzmann method with a slip boundary, this paper simulates Couette flow at a mesoscopic scale and calculates the boundary slip length on superhydrophobic surfaces at various temperatures. The agreement of experimental and numerical results suggests the effectiveness of the current method for boundary slip problems. Furthermore, the results showed that slip length on a superhydrophobic surface boundary is above $100 \mu \mathrm{m}$ at mesoscopic scale, and the surface friction is a lot less than with a non-slip boundary. The relationship between slip length and viscosity, contact angle, and velocity gradients in Couette flow is also examined. Numerical results show that the main factors affecting slip length are viscosity and contact angle. Velocity gradient has little effect on slip length.
\end{abstract}

KEYWORDS: slip length, superhydrophobic surface, resistance reduction

MSC2010: 76E05 76M28 35Q20 06B75 82D15

\section{INTRODUCTION}

The assumption of a non-slip boundary has been proved to be correct for macroscale flow. However, studies show that the fluid near the boundary can slip in the case of micro-scale flow ${ }^{1,2}$ and a clear boundary slip phenomenon can be observed at a smaller scale ${ }^{3}$. Compared with the inertial force and the electromagnetic force, the surface tension and the friction increase greatly with the decrease of scale. Since boundary slip has a vital influence on friction at micro-scale, the topic of reducing friction by producing boundary slip has attracted a lot of attention recently. Some researchers have shown that friction can be reduced when the liquid flows on a superhydrophobic surface due to the boundary slip phenomenon ${ }^{4,5}$. The boundary slip phenomenon is affected by many factors such as surface roughness ${ }^{6}$, fluid viscosity ${ }^{7}$, contact angle ${ }^{8}$, and velocity gradient ${ }^{9}$. There is no final conclusion about the effect of those factors. This reflects the complexity of the boundary slip phenomenon.

Priezjev, using the molecular dynamics method, studied the impact of shear rate and other factors on slip length and friction for boundary slip prob- lems at microscale ${ }^{10,11}$. At nanometre-scale, Priezjev's simulation is quite successful ${ }^{12}$. Simulating flow with boundary slip at millimetre scale is more difficult. Based on the mesoscopic kinetic mode, the lattice Boltzmann method (LBM) is suitable for mesoscopic simulations whose non-continuous nature fits the actual fluid. Moreover, it has a natural advantage of high calculation efficiency which can be parallelized. Nourmohammadzadeh applied the lattice Boltzmann method to simulate boundary slip phenomena on the gas-solid boundary ${ }^{13}$. Sbragalia studied the phenomenon of solid-liquid boundary slip $^{14}$. Zhu implemented three-dimensional simulations for boundary slip problems ${ }^{15}$. Their studies are all limited to microscale. Guo proposed a gas boundary slip boundary condition ${ }^{16}$. Zhang ${ }^{17}$ and $\mathrm{Chen}^{18}$ used the same method to calculate the micro-scale boundary slip length, but their simulations do not indicate the relationship between the various physical factors and slip length. Hence, by combining the methods of Guo and Zhang, this paper paper studies the effect of temperature, velocity gradients, fluid viscosity, and contact angle on the slip length of flow on a superhydrophobic surface at mesoscopic scale. The main content of 
this paper is to adopt the lattice Boltzmann method to simulate boundary slip phenomenon at mesoscale and to calculate the slip length.

\section{NUMERICAL MODEL}

Meeting the required simulation accuracy, the simple and stable LBGK model is chosen. Its evolution equation can be expressed as

$$
\begin{aligned}
f_{i}\left(\mathbf{x}+\mathbf{c}_{i} \Delta t, t+\Delta t\right)- & f_{i}(\mathbf{x}, t)= \\
& -\frac{1}{\tau}\left[f_{i}(\mathbf{x}, t)-f_{i}^{(\mathrm{eq})}(\mathbf{x}, t)\right]
\end{aligned}
$$

where $f_{i}(\mathbf{x}, t)$ is the velocity distribution function of the point $\mathbf{x}$ at time $t$, which represents the number of particles moving with velocity $\mathbf{c}_{i}$. The dimensionless relaxation time,

$$
\tau=\frac{v}{c_{s}^{2} \delta_{t}}+\frac{1}{2}
$$

$\delta_{t}$ is the time step, $f_{i}^{(\mathrm{eq})}$ is the particle equilibrium distribution function (DF). For the standard D2Q9 model, the equilibrium distribution function (EDF) can be expressed as

$$
f_{i}(\rho, \mathbf{u})=\omega_{i} \rho\left[1+\frac{\mathbf{c}_{i} \cdot \mathbf{u}}{c_{s}^{2}}+\frac{\left(\mathbf{c}_{i} \cdot \mathbf{u}\right)^{2}}{2 c_{s}^{4}}-\frac{\mathbf{u}^{2}}{2 c_{s}^{2}}\right]
$$

and we have

$$
\begin{gathered}
\mathbf{c}_{i}= \begin{cases}(0,0), & i=0, \\
\left(\cos \frac{(i-1) \pi}{2}, \sin \frac{(i-1) \pi}{2}\right) c, & i=1,2,3,4, \\
\sqrt{2}\left(\cos \frac{(2 i-1) \pi}{4}, \sin \frac{(2 i-1) \pi}{4}\right) c, & i=5,6,7,8,\end{cases} \\
\omega_{i}= \begin{cases}1, & i=0, \\
\frac{1}{9}, & i=1,2,3,4, \\
\frac{1}{36}, & i=5,6,7,8 .\end{cases}
\end{gathered}
$$

The density and velocity expressions at macroscopic scale are

$$
\rho=\sum_{i} f_{i}, \quad \rho \mathbf{u}=\sum_{i} \mathbf{c}_{i} f_{i}, c_{s}=\frac{c}{\sqrt{3}} .
$$

For different kinds of fluids, the main factor affecting the flow field at micro-scale is different. The gas flow is controlled by its characteristics of rarefaction. For liquid flow one should consider the effect of surface force, intermolecular forces, electrostatic force and invasion/adsorption characteristics. Currently, there are two boundary conditions for gas flow: BSR model and DM model ${ }^{16}$. Their collision step is the same but their stream step is different.
Because of its widely applicability, the BSR model is used here. The BSR model collision step is

$$
f_{i}^{\prime}=f_{i}+\frac{1}{\tau}\left[f_{i}-f_{i}^{(\mathrm{eq})}\right]+\delta_{t} F_{i} .
$$

and its stream step is

$$
\left\{\begin{array}{l}
f_{2}=f_{4}{ }^{\prime}, \\
f_{5}=r f_{7}^{\prime}+(1-r) f_{8}^{\prime}+2 r \rho \omega_{i} \cdot \mathbf{c}_{5} \mathbf{u}_{\omega} / 2 c_{s}^{2}, \\
f_{6}=r f_{8}^{\prime}+(1-r) f_{7}^{\prime}+2 r \rho \omega_{i} \cdot \mathbf{c}_{6} \mathbf{u}_{\omega} / 2 c_{s}^{2} .
\end{array}\right.
$$

For $i=0,1,3,7,8$, the particle distribution function can be calculated according to the transfer step. We found that $r=0.007$ is suitable for this simulation. The most important thing is to select the adhesion retentive force. The latest research shows that the adhesion force is connected with advancing contact angle, receding contact angle, surface tension, and the radius of a wetting droplet ${ }^{18}$.

$$
F=\pi R \gamma \sin \theta\left(\cos \theta_{\mathrm{r}}-\cos \theta_{\mathrm{a}}\right)
$$

where $R$ is the radius of wetting droplet, $\gamma$ is the surface tension, $\theta_{\mathrm{a}}$ is the advancing contact angle, $\theta_{\mathrm{r}}$ is the receding contact angle, and $\theta=\frac{1}{2}\left(\theta_{\mathrm{a}}+\theta_{\mathrm{r}}\right)$. According to the external force model proposed by Guo $^{16}$, the evolution equation of LBM with external force can be expressed as

$$
\begin{aligned}
f_{i}\left(\mathbf{x}+\mathbf{c}_{i} \Delta t, t+\Delta t\right)=f_{i}(\mathbf{x}, t) \\
\quad-\frac{1}{\tau}\left[f_{i}(\mathbf{x}, t)-f_{i}^{(\mathrm{eq})}(\mathbf{x}, t)\right]+F_{i}(\mathbf{x}, t) \Delta t .
\end{aligned}
$$

$F_{i}(\mathbf{x}, t)$ is the discrete external force $F(\mathbf{x}, t)$, and the discrete force distribution function is

$$
\begin{aligned}
F_{i}(\mathbf{x}, t) & =\left(1-\frac{1}{2 \tau}\right) \omega_{i} \\
& \times\left[\frac{\mathbf{c}_{i}-\mathbf{u}(\mathbf{x}, t)}{c_{s}^{2}}+\frac{\mathbf{c}_{i} \cdot \mathbf{u}(\mathbf{x}, t)}{c_{s}^{4}}\right] \cdot \mathbf{F}(\mathbf{x}, t) .
\end{aligned}
$$

However, the velocity expression in this function must take into account the influence of the external force, and it can be expressed as

$$
\rho \mathbf{u}=\sum_{i} \mathbf{c}_{i} f_{i}+\frac{\Delta t}{2} \mathbf{F} .
$$

\section{NUMERICAL MODEL VALIDATION}

We performed a non-slip 2-D Couette flow simulation to verify the correctness of the program. The size of the actual flow field is $0.3 \times 1.2 \mathrm{~cm}^{2}$. The upper plate moves at a constant speed $U_{0}=$ 


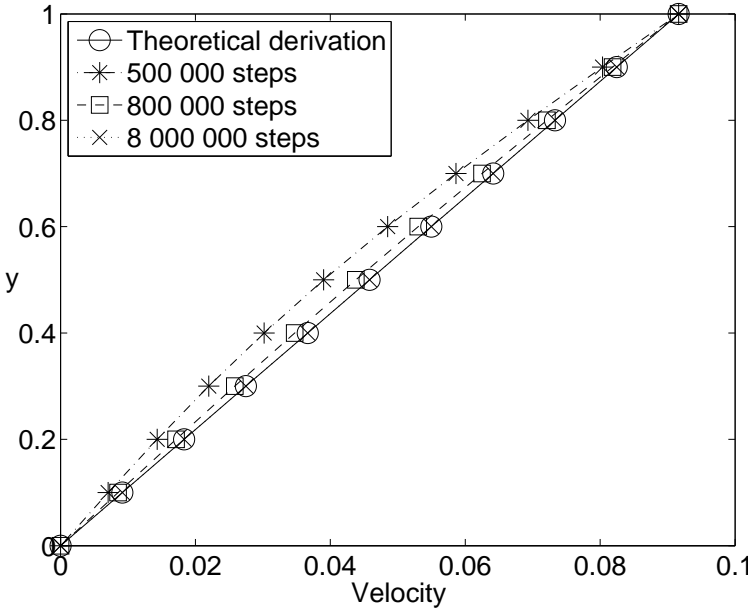

Fig. 1 The velocity distribution of a non-slip boundary at different times with LBM simulation. $y=h / H$ where $H$ is the gap between the two plates and $h$ is the distance from fluid point to the bottom plate and the velocity is dimensionless speed in the LBM.

$0.36652 \mathrm{~m} / \mathrm{s}$. The flow field is divided into a $60 \times 480$ grid. The upper plate moves at a constant speed $u=0.09163$. The upper and lower boundaries are non-equilibrium extrapolation boundaries and the left and right boundaries are subject to periodic boundary conditions. The simulation result is fairly consistent with the theoretical solution based on the Navier-Stokes equations. As is shown in Fig. 1, the velocity distribution shows little difference between the simulation result and the theoretical solution up to 8000000 time steps (corresponding to a real time of $5 \mathrm{~s}$ ). The agreement decreases with increasing time after this.

We define the friction factor as

$$
C=\frac{F}{\frac{1}{2} \rho U^{2} L} \text {. }
$$

The simulation result is $C_{s}=0.045461$ and the theoretical result is $C_{t}=0.045609$. The relative error is $0.3 \%$. According to Newton's law of internal friction, friction is also falls with the decrease of velocity gradient. This explains why the friction coefficient of simulation is less than the theoretical result. From that case, we conclude that we can simulate the Couette flow using the lattice Boltzmann method.

We need change boundary condition on the bottom plate for flow with boundary slip. As introduced above, the BSR model is used for the boundary slip simulation. The reduced retentive force is considered as the force acting on the fluid layer close to
Table 1 The advancing and receding angle for different materials.

\begin{tabular}{lcccccc}
\hline material & & 1 & 2 & 3 & 4 & 5 \\
\hline $20^{\circ} \mathrm{C}$ & $\theta_{\mathrm{a}}$ & $152.7^{\circ}$ & $153.7^{\circ}$ & $156.3^{\circ}$ & $159.3^{\circ}$ & $164.5^{\circ}$ \\
& $\theta_{\mathrm{r}}$ & $0.0^{\circ}$ & $87.3^{\circ}$ & $102.3^{\circ}$ & $127.0^{\circ}$ & $160.0^{\circ}$ \\
\hline $50^{\circ} \mathrm{C}$ & $\theta_{\mathrm{a}}$ & $157.7^{\circ}$ & $158.5^{\circ}$ & $160.6^{\circ}$ & $162.3^{\circ}$ & $162.7^{\circ}$ \\
& $\theta_{\mathrm{r}}$ & $124.7^{\circ}$ & $126.3^{\circ}$ & $146.0^{\circ}$ & $150.0^{\circ}$ & $156.5^{\circ}$ \\
\hline
\end{tabular}

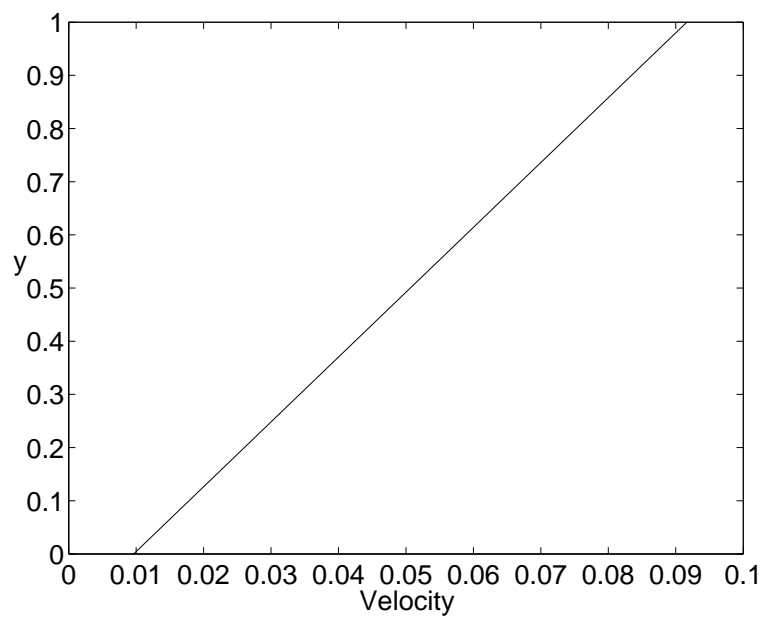

Fig. 2 The velocity distribution with boundary slip. The temperature is $20^{\circ} \mathrm{C}$.

the plate. In order to verify convergence of grid, we divide the flow field into $30 \times 480,60 \times 480$, and $120 \times 480$ grids. The simulation results show that the slip lengths are almost the same. After 3000000 time steps, the velocity distributions and the slip lengths are the same.

\section{SIMULATION RESULTS}

Table 1 shows the advancing angle and receding angle of different materials. We used a temperature of $20^{\circ} \mathrm{C}, \gamma=0.0728 \mathrm{~N} / \mathrm{m}, R=1.125 \times 10^{-5} \mathrm{~m}$, $\theta_{\mathrm{a}}=164.5^{\circ}$, and $\theta_{\mathrm{r}}=160.0^{\circ}$. The grid is $60 \times 480$. In lattice units, $F=0.1878, \tau=0.8009$. Fig. 2 shows the velocity distribution along the $y$-axis. It can be seen clearly that the velocity at the bottom is 0.00952 instead of 0 . The drag coefficient $C_{\mathrm{S}}$ is 0.040556 , which is $11 \%$ less than for the nonslip case. The slip length of that simulation can be calculated and is $139.1 \mu \mathrm{m}$. The slip length $L_{\mathrm{s}}$ can be also estimated by

$$
\frac{\tau_{\text {slip }}}{\tau_{\text {no-slip }}}=\frac{1}{1+\frac{L_{s}}{H}}
$$




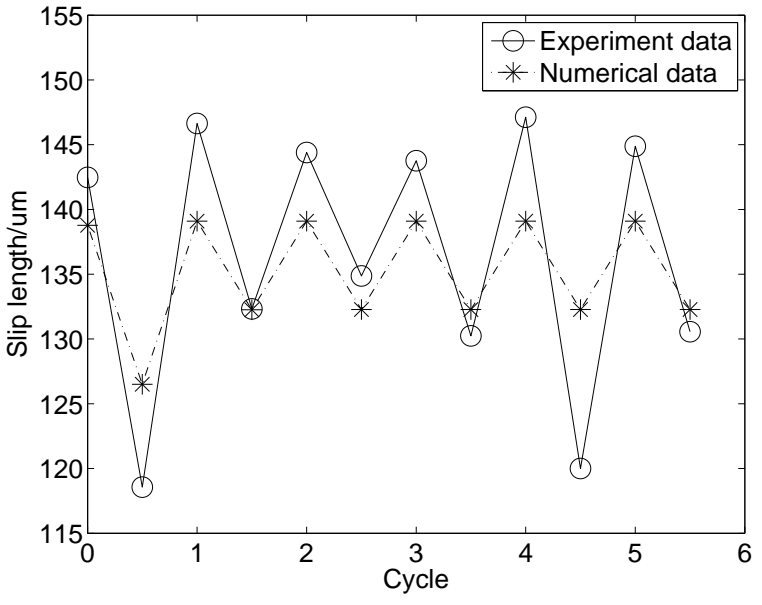

Fig. 3 The change of slip length with the change of temperature. The temperature is changed from $20^{\circ} \mathrm{C}$ to $50^{\circ} \mathrm{C}$ cyclically.

where $\tau_{\text {slip }}$ and $\tau_{\text {no-slip }}$ are the shear stresses at the wall with slip and non-slip boundary conditions. In the experiment, the slip length is $142.4 \mu \mathrm{m}$.

\section{Influence of temperature on the slip length}

The most significant factor affecting slip length is the temperature which has an important impact on the viscosity and the wettability of the boundary. We periodically change the temperature from $20^{\circ} \mathrm{C}$ to $50^{\circ} \mathrm{C}$ and observe the changes of slip length with the temperature. Fig. 3 shows the contrast of numerical and experimental results. The higher point is the slip length at $20^{\circ} \mathrm{C}$, and the lower point is the slip length at $50^{\circ} \mathrm{C}$. The average slip length of simulation and experiment are close while the fluctuation in the experiment is larger than in the simulation. With more confounding factors in the experiment, such as stability of the motor, this to be expected. When the temperature is changed, two important factors are changed, which are contact angle and viscosity.

\section{Influence of viscosity on the slip length}

Assuming there is no boundary slip, the friction increases linearly with viscosity according to Newton's law of internal friction. However, the slip length has little effect. We conclude from Fig. 4 that the slip length reduces when viscosity increases, but not in a linear manner. The slip length with the contact angle $\theta_{1}=159.6^{\circ}$ (the temperature is $50^{\circ} \mathrm{C}$ ) is smaller than the slip length with the contact angle $\theta_{2}=162.3^{\circ}$ (the temperature is $20^{\circ} \mathrm{C}$ ) no matter how the viscosity is changed. What should be emphasized is that only the viscosity is changed

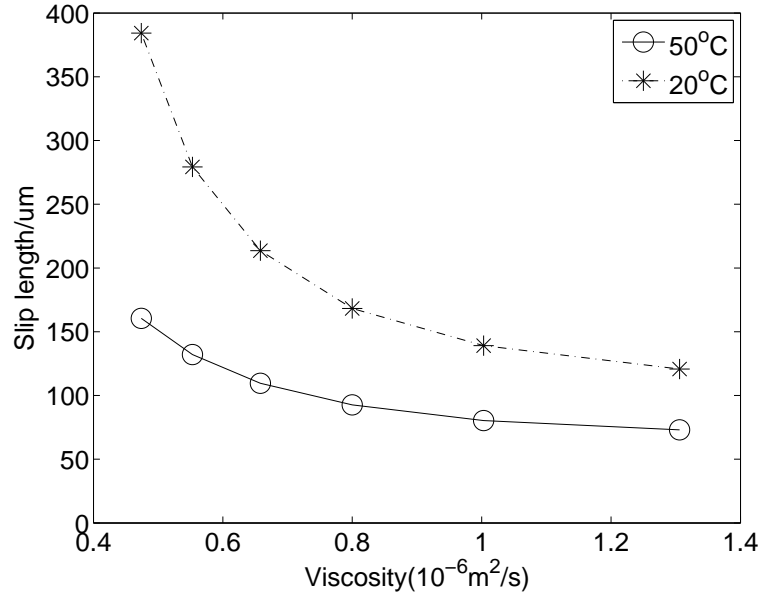

Fig. 4 Variation of slip length with viscosity. The material is no. 5 .

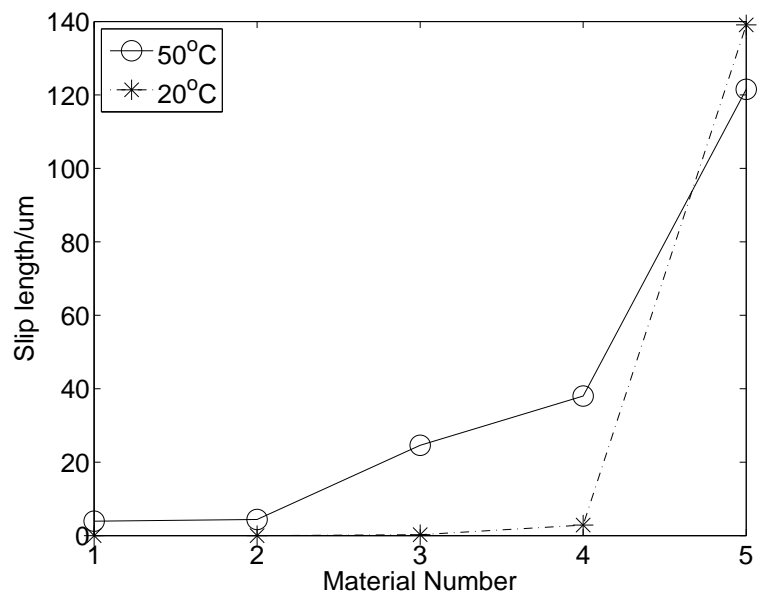

Fig. 5 Effect of material on slip length. The details of the materials are in Table 1.

here. Hence this situation cannot be examined by experiment.

\section{Influence of contact angle on the slip length}

In Fig. 5, $v_{1}$ and $v_{2}$ are the viscosities of the water at $50^{\circ} \mathrm{C}$ and $20^{\circ} \mathrm{C}$, respectively. The slip length decreases markedly as the contact angle is reduced. When the contact angle is smaller than $150^{\circ}$, there is a no boundary slip phenomenon. We conclude that boundary slip occurs only on the superhydrophobic surface. From Table 1, the contact angle at $20^{\circ} \mathrm{C}$ of material no. 5 is the biggest, while its slip length is the largest. The slip length decreases as the contact angle decreases. This is consistent with Bonaccurso's view ${ }^{3}$. 


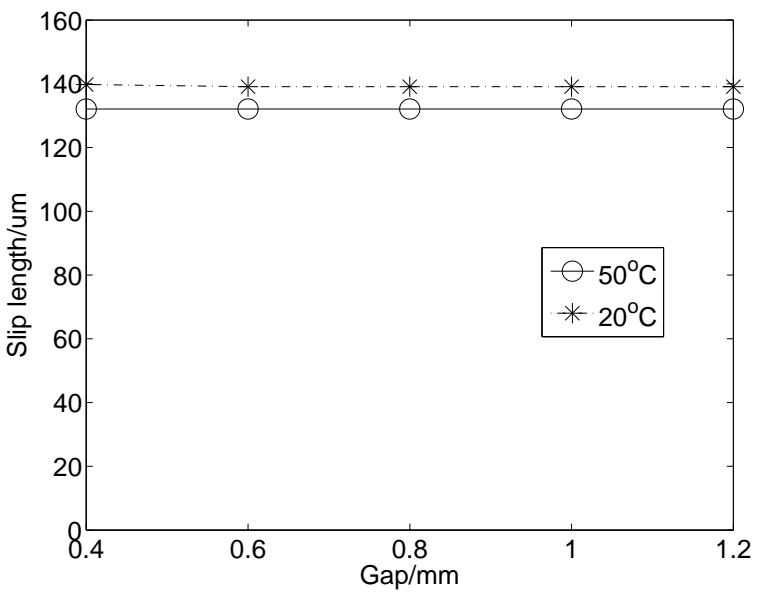

Fig. 6 The boundary slip length at different gaps.

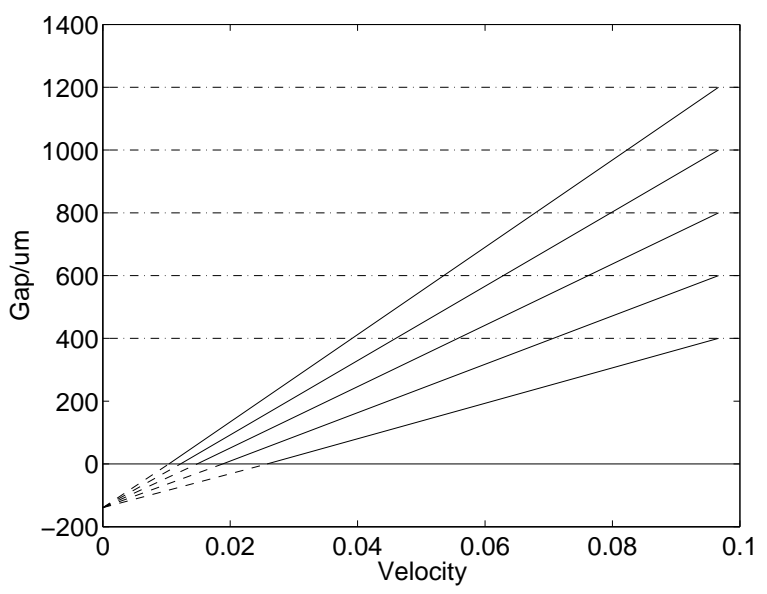

Fig. 7 Velocity distributions in different gaps.

\section{Influence of gaps on the slip length}

The velocity gradient is dependent on the gap, where the velocity of plate is constant. When the gap is small, the resistance increases with large velocity gradient. However, the boundary slip length does not significantly change, which is shown in Fig. 6 . The slip velocity is closely related with the gap, which can be seen in Fig. 7. The rules of boundary slip length changes are different from rules of the velocity gradient changes. The boundary slip length is constant according to Navier's model of linear slip length ${ }^{19}$. This conclusion is also consistent with the experiment results $6,9,20$.

\section{CONCLUSIONS}

In this paper, the lattice Boltzmann method and BSR model of boundary conditions are used to study the phenomena relating to boundary slip on superhydrophobic surface. The main factors affecting slip length and their influence on friction are investigated and the following conclusions can be made. Boundary slip occurs on a superhydrophobic boundary even at mesoscopic scales. The slip length can be above $100 \mu \mathrm{m}$. Contact angle, advancing angle, and receding angle have a great effect on slip length. A large contact angle with little difference between advancing angle and receding angle results in a larger slip length. Velocity gradient has little influence on boundary slip length, while the speed at the boundary is greatly changed. A small viscosity leads to a large slip velocity and a small contact angle results in a small slip velocity. The combined effect of both reflects the effect of temperature on the slip length.

Acknowledgements: We acknowledge financial support from the National Natural Science Foundation of China (grant no. 51475179) and the Foundation of Huazhong University of Science and Technology.

\section{REFERENCES}

1. Tretheway DC, Meinhart CD (2002) Apparent fluid slip at hydrophobic microchannel walls. Phys Fluids 14, 9-12.

2. Baudry J, Charlaix E (2001) Experimental evidence for a large slip effect at a nonwetting fluid-solid interface. Langmuir 17, 5232-6.

3. Bonaccurso E, Butt HJ, Craig VSJ (2003) Surface roughness and hydrodynamic boundary slip of a Newtonian fluid in a completely wetting system. Phys Rev Lett 90, 144501.

4. Chini SF, Bertola V, Amirfazli A (2013) A methodology to determine the adhesion force of arbitrarily shaped drops with convex contact lines. Colloid Surf A 436, 425-33.

5. Daniello RJ, Waterhouse NE, Rothstein JP (2009) Drag reduction in turbulent flows over superhydrophobic surfaces. Phys Fluids 21, 085103.

6. Vinogradova OI, Yakubov GE (2003) Dynamic effects on force measurements. 2. Lubrication and the atomic force microscope. Langmuir 19, 1227-34.

7. Vinogradova OI (1998) Implications of hydrophobic slippage for the dynamic measurements of hydrophobic forces. Langmuir 14, 2827-37.

8. Bonaccurso E, Kappl M, Butt HJ (2002) Hydrodynamic force measurements: Boundary slip of water on hydrophilic surfaces and electrokinetic effects. Phys Rev Lett 88, 076103.

9. Joseph P, Tabeling P (2004) Direct measurement of the apparent slip length. Phys Rev E 71, 035303.

10. Niavarani A, Priezjev NV (2010) Modeling the combined effect of surface roughness and shear rate on slip flow of simple fluids. Phys Rev E 81, 011606. 
11. Priezjev NV (2011) Molecular diffusion and slip boundary conditions at smooth surfaces with periodic and random nanoscale textures. $J$ Chem Phys 135, 204704.

12. Priezjev NV (2009) Shear rate threshold for the boundary slip in dense polymer films. Phys Rev E 80, 031608.

13. Nourmohammadzadeh M, Rahnama M, Jafari S, Akhgar AR (2011) Microchannel flow simulation in transition regime using lattice Boltzmann method. Proc IME C J Mech Eng Sci 226, 552-62.

14. Sbragaglia M, Benzi R, Biferale L, Succi S, Toschi F (2006) Surface roughness hydrophobicity coupling in microchannel and nanochannel flows. Phys Rev Lett 97, 204503.

15. Zhu LD, Tretheway D, Petzold L, Meinhart C (2005) Simulation of fluid slip at 3D hydrophobic microchannel walls by the lattice Boltzmann method. J Comput Phys 202, 181-95.

16. Guo ZL, Zheng CG (2009) Theory and Application of Lattice Boltzmann Method, Science Press, Beijing.

17. Zhang RL, Di QF, Wang XL, Ding WP, Gong W (2012) Numerical study of the relationship between apparent slip length and contact angle by lattice Boltzmann method. $J$ Hydrodyn B 24, 535-40.

18. Chen YY, Yi HH, Li HB (2008) Boundary slip and surface interaction: a lattice Boltzmann simulation. Chin Phys Lett 25, 184-7.

19. Vinogradova OI (1995) Drainage of a thin liquid film confined between hydrophobic surfaces. Langmuir 11, 2213-20.

20. Pit R, Hervet H, Léger L (2000) Direct experimental evidence of slip in hexadecane: solid interfaces. Phys Rev Lett 85, 980-3. 